\section{Commentary: Standardization of procedures for health care providers safety in the coronavirus disease 2019 (COVID-19) era, with an eye to the future}

\author{
Marco Scarci, MD, FRCS(Eng), FCCP, FACS, \\ FEBTS, and Federico Raveglia, MD
}

This article stimulated our interest for 2 reasons, as it reports both a pathway to safely perform tracheostomy and, more generally, suggests a model for surgical practice during and after the coronavirus disease 2019 (COVID-19) era. ${ }^{1}$ In the pandemic scenario, a tracheotomy is frequently indicated for patients affected by COVID-19 and severe acute respiratory syndrome. To our knowledge, guidelines showing the appropriate timing to perform a tracheostomy are not available and, also, waiting for a negative throat swab is not always feasible, especially during an emergency. Since tracheotomy exposes the airway, surgeons are directly exposed to the droplets and aerosol particles generated by the cough reflex, a procedure that carries one of the greatest risks of virus transmission.

Thal and coworkers ${ }^{2}$ have already reported a case series of 65 procedures in which they showed that infection risk for surgical providers is minimal if standard protective equipment is adopted (surgical gown, gloves, glasses or shield, hair cap, and N95 mask). Lee and coworkers ${ }^{3}$ focused on a protocol to protect surgeons and guarantee patient safety, considering that communication between staff members wearing personal protective equipment is challenging and, if an emergency happens, this cannot be

\footnotetext{
From the Department of Thoracic Surgery, ASST Monza e Brianza, Ospedale San Gerardo, Monza, Italy.

Disclosures: The authors reported no conflicts of interest.

The Journal policy requires editors and reviewers to disclose conflicts of interest and to decline handling or reviewing manuscripts for which they may have a conflict of interest. The editors and reviewers of this article have no conflicts of interest.

Received for publication Nov 29, 2020; revisions received Nov 29, 2020; accepted for publication Dec 15, 2020; available ahead of print Dec 26, 2020.

Address for reprints: Marco Scarci, MD, FRCS(Eng), FCCP, FACS, FEBTS, ASST

Monza, Via Pergolesi 33, Monza, Italy (E-mail: marco.scarci@mac.com).

JTCVS Techniques 2021;6:188-9

2666-2507

Copyright (C) 2021 The Authors. Published by Elsevier Inc. on behalf of The American Association for Thoracic Surgery. This is an open access article under the CC BY license (http://creativecommons.org/licenses/by/4.0/)

https://doi.org/10.1016/j.xjtc.2020.12.028
}

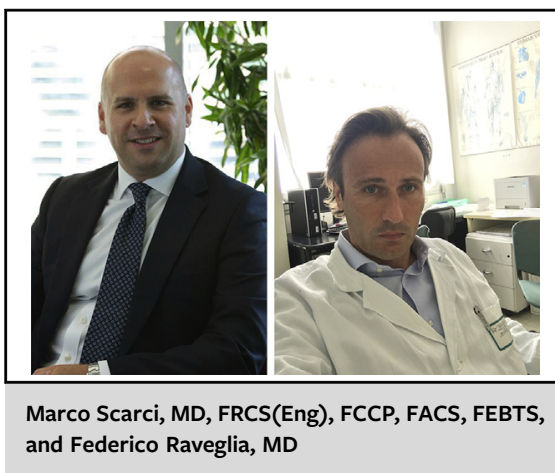

\begin{abstract}
CENTRAL MESSAGE
Multidisciplinary approach and standardization of procedures deliver an improvement to patients and healthcare providers during COVID-19, but lessons can be learnt to enhance services after pandemia.
\end{abstract}

appropriately managed unless all necessary devices are prepared in advance.

In this article, instead, the authors have remarkably emphasized that infective risk is a matter that concerns all operators who take part in the procedure and have adopted a multidisciplinary approach to determine a panel of recommendations aimed to protect all health care professionals. Interestingly, their method is based on a laboratory simulation and on a checklist-based approach.

It is our opinion that in modern medicine, patient management requires a multidisciplinary approach and standardization of procedures to guarantee the safety of operators and good outcomes for patients while saving resources at the same time. The advent of the pandemic has made these topics even more pressing. Therefore, starting from this manuscript, we would like to remark on 2 main issues. First of all, we suggest that the authors' method could be adopted to create guidelines for other invasive procedures in COVID-19 patient management. In particular, we refer to chest tube insertion, a procedure that is performed at the bedside on the ward or in the intensive care unit for pleural effusions or, more often, positive pressure-induced pneumothorax. In some studies, authors have already faced the issue of chest drain aerosol generation and COVID-19 infection. ${ }^{4}$ Second, we prompt readers to consider the unique opportunity offered by the pandemic to rethink 
clinical pathways to deliver service improvements and greater efficiency during the difficult recovery phase. To conclude, this paper is an excellent example of workflow reorganization during an emergency and for the upcoming periods after the pandemia.

\section{References}

1. Bribriesco AC, Sudarshan M, Gillespie CT, Bryson PC, Hopkins B, Tanner D, et al. Coronavirus disease 2019 (COVID-19): team preparation and approach to tracheostomy. Guidelines and recommendations from Cleveland Clinic COVID19 tracheostomy working group. J Thorac Cardiovasc Surg Tech. 2021;6:183-7.

2. Thal AG, Schiff BA, Ahmed Y, Cao A, Mo A, Mehta V, et al. Tracheotomy in a high-volume center during the COVID-19 pandemic: evaluating the surgeon's risk. Otolaryngol Head Neck Surg. September 1, 2020 [Epub ahead of print].

3. Lee DH, Kim S, Kim JS, Kim BG, Chang KH, Park JO. Protection of medical staff during tracheotomy: lessons learned from the COVID-19 pandemic. ORL J Otorhinolaryngol Relat Spec. 2020;82:304-9.

4. Duffy C, Kidd A, Francis S, Tsim S, McNaughton L, Ferguson K, et al. Chest drain aerosol generation in COVID-19 and emission reduction using a simple anti-viral filter. BMJ Open Respir Res. 2020;7:e000710. 